\title{
Gradient estimates for the Fisher-KPP equation on Riemannian manifolds
}

Xin Geng and Songbo Hou*

\author{
"Correspondence: \\ housb10@163.com \\ Department of Applied \\ Mathematics, College of Science, \\ China Agricultural University, \\ Beijing, P.R. China
}

\begin{abstract}
In this paper, we consider positive solutions to the Fisher-KPP equation on complete Riemannian manifolds. We derive the gradient estimate. Using the estimate, we get the classic Harnack inequality which extends the recent result of Cao, Liu, Pendleton, and Ward (Pac. J. Math. 290(2):273-300, 2017).
\end{abstract}

MSC: $58 J 35$

Keywords: Fisher-KPP equation; Gradient estimate; Harnack inequality

\section{Introduction}

Let $(M, g)$ be a complete Riemannian manifold. We consider the parabolic equation

$$
u_{t}=\Delta u+c u(1-u)
$$

on $M \times[0, \infty)$, where $c$ is a positive constant. In the pioneering work of Fisher in 1937 [2], he proposed equation (1.1) to study the propagation of advantageous genes in a population, where $u=u(x, t)$ stands for the population density. In another well-known paper [3], Kolmogorov, Petrovsky, and Piskunov also described the solution to (1.1). Since then, the equation is often referred to as the Fisher-KPP equation and has been widely used in the study of traveling wave solutions and propagation problems (refer to [4-6] and so on).

Recently, Cao et al. [1] derived differential Harnack estimates for positive solutions to (1.1) on Riemannian manifolds with nonnegative Ricci curvature. The idea comes from $[7,8]$ where a systematic method was developed to find a Harnack inequality for geometric evolution equations. In the complete noncompact case, they obtained the following theorem.

Theorem A (Cao et al.) Let $(M, g)$ be an $n$-dimensional complete noncompact Riemannian manifold with nonnegative Ricci curvature, and let $u(x, t): M \times[0, \infty) \rightarrow \mathbb{R}$ be a positive solution to (1.1), where $u$ is $C^{2}$ in $x$ and $C^{1}$ in $t$.

Let $f=\log u$, then we have

$$
\Delta f+\alpha|\nabla f|^{2}+\beta e^{f}+\phi(t) \geq 0
$$

for all $x$ and $t$, provided that

(c) The Author(s) 2018. This article is distributed under the terms of the Creative Commons Attribution 4.0 International License (http://creativecommons.org/licenses/by/4.0/), which permits unrestricted use, distribution, and reproduction in any medium, provided you give appropriate credit to the original author(s) and the source, provide a link to the Creative Commons license, and indicate if changes were made. 
(i) $0<\alpha<1$,

(ii) $\beta<\frac{-c n(1+\alpha)}{4 \alpha^{2}-4 \alpha+2 n}<0$,

(iii) $\frac{-c n(2+\sqrt{2})}{4(1-\alpha)}<\beta<\frac{-c n(2-\sqrt{2})}{4(1-\alpha)}$,

where

$$
\phi(t)=\frac{\mu\left(\frac{e^{2 \mu \omega t}}{v-\omega}-\frac{1}{\mu+\omega}\right)}{1-e^{2 \mu \omega t}}
$$

with

$$
\begin{aligned}
& \mu=\beta c \sqrt{\frac{2(1-\alpha)}{c(-c n-8 \beta(1-\alpha))}}, \\
& \nu=\left(\frac{4 \beta(1-\alpha)}{n}+c\right) \cdot \sqrt{\frac{2(1-\alpha)}{c(-c n-8 \beta(1-\alpha))}}, \\
& \omega=\sqrt{\frac{2(1-\alpha)}{n}} .
\end{aligned}
$$

Using Theorem A, one can integrate along space-time curves to get a Harnack inequality, but it is different from the classical Li-Yau Harnack [9] in form.

Gradient estimates play an important role in studying elliptic and parabolic operators. The method originated first in [10] and [11], and was further developed by Li and Yau [9], Li [12], Negrin [13], Souplet and Zhang [14], Yang [15], etc. Recent gradient estimates under the geometric flow include [16] and [17]. For more results on the nonlinear PDEs, one may refer to $[18,19]$.

In this paper, following the line in [12], we prove the following theorems.

Theorem 1.1 Let $M$ be a complete Riemannian manifold with boundary $\partial M$ (possibly empty). We denote by $B_{p}(2 R)$ the geodesic ball of radius $2 R$ around $P \in M$ not intersecting the boundary $\partial M$. Suppose that the Ricci curvature of $M$ is bounded from below by $-K(2 R)$ in $B_{p}(2 R)$, and $K(2 R) \geq 0$. Denote $K=K(2 R)$. If $u(x, t)$ is a positive smooth solution of (1.1) on $M \times[0, \infty)$, then we have

$$
\begin{aligned}
& \frac{|\nabla u|^{2}}{u^{2}}+s c(1-u)-s \frac{u_{t}}{u} \\
& \leq \frac{n s^{2}}{2(1-\varepsilon)} \frac{1}{t}+\frac{n s^{2}}{2(1-\varepsilon)(s-1)} K+\frac{s c}{q} \sqrt{\frac{n}{2(1-\varepsilon)}} M_{1} \\
& \quad+\frac{n s^{2}}{2(1-\varepsilon) R^{2}}\left(\frac{n s^{2}}{4(1-\varepsilon)(s-1)} C_{1}+(n+1) C_{1}\right. \\
& \left.\quad+C_{1} R(n-1) \sqrt{K}+C_{2}\right)
\end{aligned}
$$

on $B_{p}(R) \times(0,+\infty)$, where $C_{1}, C_{2}$ are positive constants and $0<\varepsilon<1, s>1, q>0$ such that $\frac{2(1-\varepsilon)}{n} \frac{s-1}{s q} \geq \frac{1}{\varepsilon}-1+\frac{(2 s-1)^{2}}{8}, M_{1}=\sup _{(x, t) \in B_{p}(2 R) \times[0, \infty)} u(x, t)$. In particular, we can choose $q=\frac{2(1-\varepsilon)(s-1)}{n s\left[\frac{1}{\varepsilon}-1+\frac{(2 s-1)^{2}}{8}\right]}$.

Using Theorem 1.1, we get the classic Harnack inequality. 
Theorem 1.2 Let $M$ be an n-dimensional complete noncompact Riemannian manifold with Ricci tensor $R_{i j} \geq-k g_{i j}(k \geq 0)$. If $u(x, t)$ is a positive solution of $(1.1)$ and $0<u<1$, then

$$
\begin{aligned}
u\left(x_{1}, t_{1}\right) \leq & u\left(x_{2}, t_{2}\right)\left(\frac{t_{2}}{t_{1}}\right)^{\frac{n s}{2(1-\varepsilon)}} \\
& \times \exp \left(\frac{s r^{2}}{4\left(t_{2}-t_{1}\right)}+\left(t_{2}-t_{1}\right)\left(\frac{n s k}{2(1-\varepsilon)(s-1)}+\frac{c}{q} \sqrt{\frac{n}{2(1-\varepsilon)}}\right)\right)
\end{aligned}
$$

where $x_{1}, x_{2} \in M, 0<t_{1}<t_{2}<\infty$, and $r\left(x_{1}, x_{2}\right)$ is the geodesic distance between $x_{1}$ and $x_{2}$. In particular, taking $s=3 / 2$ and $\varepsilon=1 / 4$, we get

$$
\begin{aligned}
u\left(x_{1}, t_{1}\right) \leq & u\left(x_{2}, t_{2}\right)\left(\frac{t_{2}}{t_{1}}\right)^{n} \\
& \times \exp \left(\frac{3 r^{2}}{8\left(t_{2}-t_{1}\right)}+\left(t_{2}-t_{1}\right)\left(2 n k+\frac{7 \sqrt{6} n \sqrt{n} c}{3}\right)\right) .
\end{aligned}
$$

The rest of the paper is arranged as follows. In Sect. 2, we get a technical lemma which is important to the proof. In Sect. 3, we prove Theorems 1.1 and 1.2.

\section{Technical lemma}

As in [12], we define

$$
W(x, t)=u^{-q},
$$

where $q$ is a positive constant to be fixed later. A direct computation shows that

$$
\begin{aligned}
& \nabla W=-q u^{-q-1} \nabla u, \\
& |\nabla W|^{2}=q^{2} u^{-2 q-2}|\nabla u|^{2}, \\
& \frac{|\nabla W|^{2}}{W^{2}}=q^{2} u^{-2}|\nabla u|^{2}, \\
& W_{t}=-q u^{-q-1} u_{t}, \\
& \frac{W_{t}}{W}=-q \frac{u_{t}}{u}, \\
& \begin{aligned}
\Delta W & =q(q+1) u^{-q-2}|\nabla u|^{2}-q u^{-q-1} \Delta u \\
& =\frac{q+1}{q} \frac{|\nabla W|^{2}}{W}+c q W-c q W^{\frac{q-1}{q}}+W_{t} .
\end{aligned}
\end{aligned}
$$

Therefore

$$
\left(\Delta-\frac{\partial}{\partial t}\right) W=\frac{q+1}{q} \frac{|\nabla W|^{2}}{W}+c q W-c q W^{\frac{q-1}{q}} .
$$

We follow the line in [12]. Define three functions:

$$
F_{0}(x, t)=\frac{|\nabla W|^{2}}{W^{2}}+\alpha c\left(1-W^{-1 / q}\right)
$$




$$
\begin{aligned}
& F_{1}=\frac{W_{t}}{W}, \\
& F=F_{0}+\beta F_{1},
\end{aligned}
$$

where $\alpha, \beta$ are two positive constants to be fixed later.

Let $e_{1}, e_{2}, \ldots, e_{n}$ be a local orthonormal frame field. We adopt the notation that subscripts in $i, j$, and $k$, with $1 \leq i, j, k \leq n$, denote covariant differentiations in the $e_{i}, e_{j}$, and $e_{k}$ directions, respectively.

Calculate

$$
\begin{aligned}
& \nabla F_{0}(x, t)=\frac{2 W_{i} W_{i j}}{W^{2}}-\frac{2 W_{i}^{2} W_{j}}{W^{3}}+\frac{\alpha c}{q} W^{-(q+1) / q} W_{j}, \\
& \Delta F_{0}(x, t)=\frac{2 W_{i j}^{2}}{W^{2}}+\frac{2 W_{i} W_{i j j}}{W^{2}}-8 \frac{W_{i} W_{i j} W_{j}}{W^{3}}+6 \frac{W_{i}^{4}}{W^{4}}-2 \frac{W_{i}^{2} W_{j j}}{W^{3}} \\
& -\frac{\alpha c(q+1)}{q^{2}} \frac{W^{-1 / q}}{W^{2}} W_{j}^{2}+\frac{\alpha c}{q} W^{-(q+1) / q} W_{j j}, \\
& \frac{\partial F_{0}(x, t)}{\partial t}=\frac{2 W_{i} W_{i t}}{W^{2}}-\frac{2 W_{i}^{2} W_{t}}{W^{3}}+\frac{\alpha c}{q} W^{-\frac{q+1}{q}} W_{t}, \\
& \nabla F_{1}=\frac{W_{t i} W-W_{t} W_{i}}{W^{2}} \\
& \Delta F_{1}=\frac{W W_{i i t}-2 W_{i} W_{t i}-W_{t} W_{i i}}{W^{2}}+\frac{2 W_{t} W_{i}^{2}}{W^{3}}, \\
& \frac{\partial F_{1}}{\partial t}=\frac{W_{t t} W-W_{t}^{2}}{W^{2}}, \\
& \left(\Delta-\frac{\partial}{\partial t}\right) F_{1}=\frac{W\left(\Delta W-W_{t}\right)_{t}-W_{t}\left(\Delta W-W_{t}\right)-2 W_{i} W_{t i}}{W^{2}}+\frac{2 W_{t} W_{i}^{2}}{W^{3}} \\
& =\frac{2}{q} \nabla \log W \cdot \nabla F_{1}+c W^{-\frac{1}{q}-1} W_{t} .
\end{aligned}
$$

We denote the Ricci tensor of $M$ by $R_{i j}$ :

$$
\frac{2 W_{i} W_{i j j}}{W^{2}}=\frac{2 W_{i} W_{j j i}}{W^{2}}+\frac{2 R_{i j} W_{i} W_{j}}{W^{2}} .
$$

It follows that

$$
\begin{aligned}
\frac{2 W_{i} W_{i j}}{W^{2}}-\frac{2 W_{i} W_{i t}}{W^{2}}= & \frac{2 W_{i}}{W^{2}}\left(\Delta W-W_{t}\right)_{i}+\frac{2 R_{i j} W_{i} W_{j}}{W^{2}} \\
= & \frac{4(q+1)}{q} \frac{W_{i} W_{i j} W_{j}}{W^{3}}-\frac{2(q+1)}{q} \frac{W_{i}^{4}}{W^{4}} \\
& +\frac{2 c W_{i}^{2}}{W^{2}}\left[q-(q-1) W^{-1 / q}\right]+\frac{2 R_{i j} W_{i} W_{j}}{W^{2}} .
\end{aligned}
$$

Equalities (2.2) and (2.4) yield

$$
\begin{aligned}
-\frac{2 W_{i}^{2} W_{j j}}{W^{3}}+\frac{2 W_{i}^{2} W_{t}}{W^{3}} & =-\frac{2 W_{i}^{2}}{W^{3}}\left(\Delta W-W_{t}\right) \\
& =-\frac{2(q+1)}{q} \frac{W_{i}^{4}}{W^{4}}-2 c q \frac{W_{i}^{2}}{W^{2}}+2 c q W^{-\frac{1}{q}} \frac{W_{i}^{2}}{W^{2}} .
\end{aligned}
$$


By Hölder's inequality, we have

$$
\frac{2 \varepsilon W_{i j}^{2}}{W^{2}}+\frac{2}{\varepsilon} \cdot \frac{W_{i}^{4}}{W^{4}} \geq 4 \frac{W_{i} W_{i j} W_{j}}{W^{3}} .
$$

So,

$$
\frac{2 W_{i j}^{2}}{W^{2}}-8 \frac{W_{i} W_{i j} W_{j}}{W^{3}}+6 \frac{W_{i}^{4}}{W^{4}} \geq \frac{2(1-\varepsilon) W_{i j}^{2}}{W^{2}}-4 \frac{W_{i} W_{i j} W_{j}}{W^{3}}+\left(6-\frac{2}{\varepsilon}\right) \frac{W_{i}^{4}}{W^{4}}
$$

where $0<\varepsilon<1$.

Noting the inequality $W_{i j}^{2} \geq \frac{1}{n}\left(W_{i i}\right)^{2}$, we obtain

$$
\begin{aligned}
& \frac{2 W_{i j}^{2}}{W^{2}}-8 \frac{W_{i} W_{i j} W_{j}}{W^{3}}+6 \frac{W_{i}^{4}}{W^{4}} \\
& \geq \frac{2(1-\varepsilon)}{n}\left(\frac{\Delta W}{W}\right)^{2}-4\left(\frac{W_{i} W_{i j} W_{j}}{W^{3}}-\frac{W_{i}^{4}}{W^{4}}\right)-2\left(\frac{1}{\varepsilon}-1\right) \frac{W_{i}^{4}}{W^{4}} .
\end{aligned}
$$

By (2.7), we have

$$
\nabla F_{0} \cdot \nabla \log W=\frac{2 W_{i} W_{i j} W_{j}}{W^{3}}-\frac{2 W_{i}^{4}}{W^{4}}+\frac{\alpha c}{q} W^{-\frac{1}{q}} \frac{W_{j}^{2}}{W^{2}}
$$

Plugging (2.11), (2.12), (2.13), and (2.14) into (2.8) and (2.9), we have

$$
\begin{aligned}
\left(\Delta-\frac{\partial}{\partial t}\right) F_{0} \geq & \frac{2(1-\varepsilon)}{n}\left(\frac{\Delta W}{W}\right)^{2}-2\left(\frac{1}{\varepsilon}-1\right) \frac{W_{i}^{4}}{W^{4}} \\
& +\frac{2}{q} \nabla F_{0} \cdot \nabla \log W \\
& +2 c\left(1-\frac{\alpha}{q^{2}}\right) \frac{W_{i}^{2}}{W^{2}} W^{-\frac{1}{q}} \\
& +\frac{2 R_{i j} W_{i} W_{j}}{W^{2}}+\alpha c^{2} W^{-\frac{1}{q}}-\alpha c^{2} W^{-\frac{2}{q}}
\end{aligned}
$$

Setting $\beta=\alpha / q$ and combining (2.10), we conclude that

$$
\begin{aligned}
\left(\Delta-\frac{\partial}{\partial t}\right) F \geq & \frac{2(1-\varepsilon)}{n}\left(\frac{\Delta W}{W}\right)^{2}-2\left(\frac{1}{\varepsilon}-1\right) \frac{W_{i}^{4}}{W^{4}} \\
& +\frac{2}{q} \nabla F \cdot \nabla \log W \\
& +2 c\left(1-\frac{\alpha}{q^{2}}\right) \frac{W_{i}^{2}}{W^{2}} W^{-\frac{1}{q}} \\
& +\frac{2 R_{i j} W_{i} W_{j}}{W^{2}}+\alpha c^{2} W^{-\frac{1}{q}}-\alpha c^{2} W^{-\frac{2}{q}} \\
& +\frac{\alpha c}{q} W^{-\frac{1}{q}-1} W_{t} .
\end{aligned}
$$


By (2.4) and (2.6), we arrive at

$$
\frac{\Delta W}{W}=\frac{q}{\alpha} F+\left(\frac{q+1}{q}-\frac{q}{\alpha}\right) \frac{|\nabla W|^{2}}{W^{2}} .
$$

Setting $\alpha=s q^{2}$ yields

$$
\begin{aligned}
\frac{\Delta W}{W} & =\frac{1}{s q} F+\left(\frac{q+1}{q}-\frac{1}{s q}\right) \frac{|\nabla W|^{2}}{W^{2}} \\
& =\frac{1}{s q} F+\left(\frac{q+1-1 / s}{q}\right) \frac{|\nabla W|^{2}}{W^{2}} .
\end{aligned}
$$

Substituting (2.18) into (2.16), we obtain

$$
\begin{aligned}
\left(\Delta-\frac{\partial}{\partial t}\right) F \geq & \frac{2(1-\varepsilon)}{n} \frac{1}{s^{2} q^{2}} F^{2} \\
& +\left[\frac{2(1-\varepsilon)}{n} \frac{(s q+s-1)^{2}}{s^{2} q^{2}}-2\left(\frac{1}{\varepsilon}-1\right)\right] \frac{|\nabla W|^{4}}{W^{4}} \\
& +\frac{4(1-\varepsilon)}{n} \frac{(s q+s-1)}{s^{2} q^{2}} F \frac{|\nabla W|^{2}}{W^{2}}+\frac{2}{q} \nabla F \cdot \nabla \log W \\
& +2 c(1-s) \frac{|\nabla W|^{2}}{W^{2}} W^{-\frac{1}{q}}+\frac{2 R_{i j} W_{i} W_{j}}{W^{2}}+s q^{2} c^{2} W^{-\frac{1}{q}} \\
& -s q^{2} c^{2} W^{-\frac{2}{q}}+s q c W^{-\frac{1}{q}-1} W_{t} .
\end{aligned}
$$

An immediate consequence is the following lemma.

Lemma 2.1 Let $M$ be an n-dimensional complete Riemannian manifold with Ricci tensor $R_{i j}$. If $F$ is defined by (2.6) where $\beta=\alpha / q, \alpha=s q^{2}$, then we have

$$
\begin{aligned}
\left(\Delta-\frac{\partial}{\partial t}\right) F \geq & \frac{2(1-\varepsilon)}{n} \frac{1}{s^{2} q^{2}} F^{2} \\
& +\left[\frac{2(1-\varepsilon)}{n} \frac{(s q+s-1)^{2}}{s^{2} q^{2}}-2\left(\frac{1}{\varepsilon}-1\right)\right] \frac{|\nabla W|^{4}}{W^{4}} \\
& +\frac{4(1-\varepsilon)}{n} \frac{(s q+s-1)}{s^{2} q^{2}} F \frac{|\nabla W|^{2}}{W^{2}} \\
& +\frac{2}{q} \nabla F \cdot \log W+(c-2 c s) \frac{|\nabla W|^{2}}{W^{2}} W^{-\frac{1}{q}} \\
& +c W^{-\frac{1}{q}} F+\frac{2 R_{i j} W_{i} W_{j}}{W^{2}} .
\end{aligned}
$$

\section{Main theorems}

Theorem 3.1 Let $M$ be a complete Riemannian manifold with boundary $\partial M$ (possibly empty). We denote by $B_{p}(2 R)$ the geodesic ball of radius $2 R$ around $P \in M$ not intersecting the boundary $\partial M$. Suppose that the Ricci curvature of $M$ is bounded from below by $-K(2 R)$ in $B_{p}(2 R)$, and $K(2 R) \geq 0$. Denote $K=K(2 R)$. If $u(x, t)$ is a positive smooth solution of $(1.1)$ 
on $M \times[0, \infty)$, then we have

$$
\begin{aligned}
& \frac{|\nabla u|^{2}}{u^{2}}+s c(1-u)-s \frac{u_{t}}{u} \\
& \leq \frac{n s^{2}}{2(1-\varepsilon)} \frac{1}{t}+\frac{n s^{2}}{2(1-\varepsilon)(s-1)} K+\frac{s c}{q} \sqrt{\frac{n}{2(1-\varepsilon)}} M_{1} \\
& \quad+\frac{n s^{2}}{2(1-\varepsilon) R^{2}}\left(\frac{n s^{2}}{4(1-\varepsilon)(s-1)} C_{1}+(n+1) C_{1}\right. \\
& \left.\quad+C_{1} R(n-1) \sqrt{K}+C_{2}\right)
\end{aligned}
$$

on $B_{p}(R) \times(0,+\infty)$, where $C_{1}, C_{2}$ are positive constants, $0<\varepsilon<1, s>1, q>0$ such that $\frac{2(1-\varepsilon)}{n} \frac{s-1}{s q} \geq \frac{1}{\varepsilon}-1+\frac{(2 s-1)^{2}}{8}$, and $M_{1}=\sup _{(x, t) \in B_{p}(2 R) \times[0, \infty)} u(x, t)$. In particular, we can choose $q=\frac{2(1-\varepsilon)(s-1)}{n s\left[\frac{1}{\varepsilon}-1+\frac{(2 s-1)^{2}}{8}\right]}$.

Proof Let $\chi \in C^{2}[0,+\infty)$ be a cut-off function such that $\chi(r)=1$ for $r \leq 1, \chi(r)=0$ for $r>2$, and $0 \leq \chi(r) \leq 1$. We choose $\chi$ satisfying $-\sqrt{C_{1}} \chi^{1 / 2}(r) \leq \chi^{\prime}(r) \leq 0, \chi^{\prime \prime}(r) \geq-C_{2}$, where $C_{1}, C_{2}$ are positive constants.

Denote by $r(x)$ the geodesic distance between $x$ and some fixed point $P$. Set

$$
\phi(x)=\chi\left(\frac{r(x)}{R}\right)
$$

By the conditions on $\chi$ and the Laplacian comparison theorem, we get

$$
|\nabla \phi|^{2} \leq \frac{C_{1}}{R^{2}} \phi
$$

and

$$
\Delta \phi \geq-\frac{C_{2}+C_{1}(n-1)}{R^{2}}-\frac{C_{1}(n-1) \sqrt{K}}{R} .
$$

Define the function $H(x, t):=t F(x, t)$. Using the argument of Calabi [20], we assume that the function $\phi(x) \cdot H(x, t)$ with support in $B_{P}(2 R)$ is smooth. For any fixed $T>0$, let $\left(x_{0}, t_{0}\right)$ be the point where $\phi \cdot H$ achieves its maximum in $B_{P}(2 R) \times[0, T]$. Without loss of generality, we assume that $\phi\left(x_{0}\right) \cdot H\left(x_{0}, t_{0}\right)>0$. Otherwise, (3.1) is obviously true. By the maximum principle, at $\left(x_{0}, t_{0}\right)$, we have

$$
\begin{aligned}
& \nabla(\phi \cdot H)=0, \\
& \frac{\partial(\phi \cdot H)}{\partial t} \geq 0, \\
& \Delta(\phi \cdot H) \leq 0 .
\end{aligned}
$$

By (3.2), we have

$$
\nabla H=-\frac{\nabla \phi}{\phi} H
$$


By (3.4), we have

$$
\Delta \phi \cdot H+2 \nabla \phi \cdot \nabla H+\phi \Delta H \leq 0 .
$$

It follows from (3.3) and (3.6) that

$$
\Delta \phi \cdot H+2 \nabla \phi \cdot \nabla H+\phi\left(\Delta-\frac{\partial}{\partial t}\right) H \leq 0
$$

Setting $\beta=\alpha / q, \alpha=s q^{2}$, by Lemma 2.1 we have

$$
\begin{aligned}
\left(\Delta-\frac{\partial}{\partial t}\right) H= & t\left(\Delta-\frac{\partial}{\partial t}\right) F-F \\
\geq & \frac{2(1-\varepsilon)}{n} \frac{1}{s^{2} q^{2}} H^{2} \frac{1}{t}+\left[\frac{2(1-\varepsilon)}{n} \frac{(s q+s-1)^{2}}{s^{2} q^{2}}-2\left(\frac{1}{\varepsilon}-1\right)\right] \frac{|\nabla W|^{4}}{W^{4}} t \\
& +\frac{4(1-\varepsilon)}{n} \frac{(s q+s-1)}{s^{2} q^{2}} \frac{|\nabla W|^{2}}{W^{2}} H+\frac{2}{q} \nabla H \cdot \nabla \log W+c W^{-\frac{1}{q}} H \\
& -c(2 s-1) \frac{|\nabla|^{2}}{W^{2}} W^{-1 / q} t-2 K \frac{|\nabla W|^{2}}{W^{2}} t-\frac{H}{t} .
\end{aligned}
$$

By Hölder's inequality, we get

$$
2 K t \frac{|\nabla W|^{2}}{W^{2}} \leq \frac{2(1-\varepsilon)}{n} \frac{(s-1)^{2}}{s^{2} q^{2}} \frac{|\nabla W|^{4}}{W^{4}} t+\frac{n}{2(1-\varepsilon)} \frac{s^{2} q^{2}}{(s-1)^{2}} K^{2} t
$$

and

$$
c(2 s-1) \frac{|\nabla W|^{2}}{W^{2}} W^{-1 / q} t \leq \frac{(2 s-1)^{2}}{4} \frac{|\nabla W|^{4}}{W^{4}} t+c^{2} M_{1}^{2} t .
$$

Substituting (3.9) and (3.10) into (3.8), and choosing $s>1$ and $q>0$ such that $\frac{2(1-\varepsilon)}{n} \frac{s-1}{s q} \geq$ $\frac{1}{\varepsilon}-1+\frac{(2 s-1)^{2}}{8}$, we have

$$
\begin{aligned}
\left(\Delta-\frac{\partial}{\partial t}\right) H \geq & \frac{2(1-\varepsilon)}{n} \frac{1}{s^{2} q^{2}} H^{2} \frac{1}{t}+\frac{4(1-\varepsilon)}{n} \frac{(s q+s-1)}{s^{2} q^{2}} \frac{|\nabla W|^{2}}{W^{2}} H \\
& +\frac{2}{q} \nabla H \cdot \nabla \log W-\frac{H}{t}-\frac{n}{2(1-\varepsilon)} \frac{s^{2} q^{2}}{(s-1)^{2}} K^{2} t-c^{2} M_{1}^{2} t .
\end{aligned}
$$

Substituting (3.11) into (3.7) and using (3.5), we have

$$
\begin{aligned}
& \frac{2(1-\varepsilon)}{n} \frac{1}{s^{2} q^{2}} H^{2} \frac{1}{t} \phi+\frac{4(1-\varepsilon)}{n} \frac{(s q+s-1)}{s^{2} q^{2}} \frac{|\nabla W|^{2}}{W^{2}} H \phi-\frac{2}{q} H \nabla \phi \cdot \frac{\nabla W}{W} \\
& -\frac{H}{t} \phi-\frac{n}{2(1-\varepsilon)} \frac{s^{2} q^{2}}{(s-1)^{2}} K^{2} \phi t-c^{2} M_{1}^{2} \phi t \\
& -\left(\frac{C_{2}+C_{1}(n+1)}{R^{2}}+\frac{C_{1}(n-1) \sqrt{K}}{R}\right) H \leq 0
\end{aligned}
$$

where we have used $2 \nabla \phi \cdot \nabla H=-2 \frac{|\nabla \phi|^{2}}{\phi} H \geq-\frac{2 C_{1}}{R^{2}} H$. 
Clearly,

$$
\begin{aligned}
& \frac{2}{q} H \nabla \phi \cdot \frac{\nabla W}{W} \\
& \quad \leq \frac{4(1-\varepsilon)}{n} \frac{(s q+s-1)}{s^{2} q^{2}} \frac{|\nabla W|^{2}}{W^{2}} H \phi+\frac{n}{4(1-\varepsilon)} \frac{s^{2} H}{(s q+s-1)} \frac{|\nabla \phi|^{2}}{\phi} .
\end{aligned}
$$

Multiplying through by $t \phi$ at (3.12) and using (3.13), we arrive at

$$
\begin{aligned}
& \frac{2(1-\varepsilon)}{n} \frac{1}{s^{2} q^{2}} H^{2} \phi^{2}-H \phi \\
& -t\left(\frac{n s^{2}}{4(1-\varepsilon)(s q+s-1)} \frac{C_{1}}{R^{2}}+\frac{C_{2}+(n+1) C_{1}}{R^{2}}+\frac{C_{1}(n-1) \sqrt{K}}{R}\right) H \phi \\
& -t^{2}\left(\frac{n}{2(1-\varepsilon)} \frac{s^{2} q^{2}}{(s-1)^{2}} K^{2}+c^{2} M_{1}^{2}\right) \leq 0 .
\end{aligned}
$$

Equation (3.14) yields

$$
\begin{aligned}
H \phi \leq & \frac{n s^{2} q^{2}}{2(1-\varepsilon)}\left[1+t\left(\frac{n s^{2}}{4(1-\varepsilon)(s q+s-1)} \frac{C_{1}}{R^{2}}+\frac{C_{2}+(n+1) C_{1}}{R^{2}}+\frac{C_{1}(n-1) \sqrt{K}}{R}\right)\right] \\
& +t \sqrt{\frac{n s^{2} q^{2}}{2(1-\varepsilon)}} \sqrt{\frac{n}{2(1-\varepsilon)} \frac{s^{2} q^{2}}{(s-1)^{2}} K^{2}+c^{2} M_{1}^{2}}
\end{aligned}
$$

at $\left(x_{0}, t_{0}\right)$.

It is easy to see that

$$
\begin{aligned}
& \sup _{x \in B_{p}(R)} H(x, T) \\
& \leq H\left(x_{0}, t_{0}\right) \phi\left(x_{0}\right) \\
& \leq \frac{n s^{2} q^{2}}{2(1-\varepsilon)}\left[1+T\left(\frac{n s^{2}}{4(1-\varepsilon)(s q+s-1)} \frac{C_{1}}{R^{2}}+\frac{C_{2}+(n+1) C_{1}}{R^{2}}+\frac{C_{1}(n-1) \sqrt{K}}{R}\right)\right] \\
&+T \sqrt{\frac{n s^{2} q^{2}}{2(1-\varepsilon)}} \sqrt{\frac{n}{2(1-\varepsilon)} \frac{s^{2} q^{2}}{(s-1)^{2}} K^{2}+c^{2} M_{1}^{2} .}
\end{aligned}
$$

Then we get

$$
\begin{aligned}
& \frac{|\nabla u|^{2}}{u^{2}}+s c(1-u)-s \frac{u_{t}}{u} \\
& \leq \frac{n s^{2}}{2(1-\varepsilon)} \frac{1}{t}+\frac{n s^{2}}{2(1-\varepsilon)(s-1)} K+\frac{s c}{q} \sqrt{\frac{n}{2(1-\varepsilon)}} M_{1} \\
& \quad+\frac{n s^{2}}{2(1-\varepsilon) R^{2}}\left(\frac{n s^{2}}{4(1-\varepsilon)(s-1)} C_{1}+(n+1) C_{1}+C_{1} R(n-1) \sqrt{K}+C_{2}\right)
\end{aligned}
$$

on $B_{p}(R) \times(0,+\infty)$ since $T>0$ is arbitrary.

Using Theorem 3.1 and letting $R \rightarrow+\infty$, we can get the following corollary. 
Corollary 3.2 Let $M$ be an n-dimensional complete Riemannian manifold with Ricci tensor $R_{i j} \geq-k g_{i j}(k \geq 0)$. If $u(x, t)$ is a positive solution of $(1.1)$ and $0<u<1$, then

$$
\frac{|\nabla u|^{2}}{u^{2}}-s \frac{u_{t}}{u} \leq \frac{n s^{2}}{2(1-\varepsilon)} \frac{1}{t}+\frac{n s^{2}}{2(1-\varepsilon)(s-1)} k+\frac{s c}{q} \sqrt{\frac{n}{2(1-\varepsilon)}} .
$$

Remark 3.3 Let $M$ be an $n$-dimensional complete Riemannian manifold with nonnegative Ricci curvature. Suppose that $u(x, t)$ is a positive solution of $(1.1)$ and $0<u<1$. Let $f=$ $\log u$. Then we have

$$
f_{t}=\Delta f+|\nabla f|^{2}+c\left(1-e^{f}\right)
$$

It follows from Corollary 3.2 that

$$
\Delta f+\frac{s-1}{s}|\nabla f|^{2}+\frac{n s}{2(1-\varepsilon)} \frac{1}{t}+\frac{c}{q} \sqrt{\frac{n}{2(1-\varepsilon)}} \geq 0 .
$$

In particular, taking $s=3 / 2$ and $\varepsilon=1 / 4$, we get

$$
\Delta f+\frac{1}{3}|\nabla f|^{2}+\frac{n}{t}+\frac{7 \sqrt{6} n \sqrt{n} c}{3} \geq 0
$$

This estimate is simpler than (1.2) in form.

Theorem 3.4 Let $M$ be an n-dimensional complete Riemannian manifold with Ricci tensor $R_{i j} \geq-k g_{i j}(k \geq 0)$. If $u(x, t)$ is a positive solution of $(1.1)$ and $0<u<1$, then

$$
\begin{aligned}
u\left(x_{1}, t_{1}\right) \leq & u\left(x_{2}, t_{2}\right)\left(\frac{t_{2}}{t_{1}}\right)^{\frac{n s}{2(1-\varepsilon)}} \\
& \times \exp \left(\frac{s r^{2}}{4\left(t_{2}-t_{1}\right)}+\left(t_{2}-t_{1}\right)\left(\frac{n s k}{2(1-\varepsilon)(s-1)}+\frac{c}{q} \sqrt{\frac{n}{2(1-\varepsilon)}}\right)\right),
\end{aligned}
$$

where $x_{1}, x_{2} \in M, 0<t_{1}<t_{2}<\infty$, and $r\left(x_{1}, x_{2}\right)$ is the geodesic distance between $x_{1}$ and $x_{2}$.

Proof If we set $f=\log u$, then

$$
|\nabla f|^{2}-s f_{t} \leq \frac{n s^{2}}{2(1-\varepsilon)} \frac{1}{t}+\frac{n s^{2}}{2(1-\varepsilon)(s-1)} k+\frac{s c}{q} \sqrt{\frac{n}{2(1-\varepsilon)}}
$$

for all $(x, t) \in M \times(0,+\infty)$.

Fix points $\left(x_{1}, t_{1}\right)$ and $\left(x_{2}, t_{2}\right)$ in $M \times(0,+\infty)$ with $t_{1}<t_{2}$, and let $r:[0,1] \rightarrow M$ be the shortest geodesic joining $x_{1}$ and $x_{2}$ with $r(0)=x_{2}$ and $r(1)=x_{1}$.

Define the curve $\eta:[0,1] \rightarrow M \times(0,+\infty)$ by $\eta(y)=\left(r(y),(1-y) t_{2}+y t_{1}\right)$. It is clear that $\eta(0)=\left(x_{2}, t_{2}\right), \eta(1)=\left(x_{1}, t_{1}\right)$ and

$$
f\left(x_{1}, t_{1}\right)-f\left(x_{2}, t_{2}\right)=\int_{0}^{1} \frac{d f(\eta(y))}{d y} d y \leq \int_{0}^{1}\left(\rho|\nabla f|-\left(t_{2}-t_{1}\right) f_{t}\right) d y
$$

where $\rho=r\left(x_{1}, x_{2}\right)$. 
By inequality (3.16), we get

$$
-f_{t} \leq-\frac{|\nabla f|^{2}}{s}+\frac{n s}{2(1-\varepsilon) t}+\frac{n s k}{2(1-\varepsilon)(s-1)}+\frac{c}{q} \sqrt{\frac{n}{2(1-\varepsilon)}} .
$$

Thus (3.17) becomes

$$
\begin{aligned}
& f\left(x_{1}, t_{1}\right)-f\left(x_{2}, t_{2}\right) \\
& \quad \leq \int_{0}^{1}\left(\rho|\nabla f|-\left(t_{2}-t_{1}\right) \frac{|\nabla f|^{2}}{s}\right. \\
& \left.\quad+\left(t_{2}-t_{1}\right) \frac{n s}{2(1-\varepsilon) t}+\left(t_{2}-t_{1}\right)\left(\frac{n s k}{2(1-\varepsilon)(s-1)}+\frac{c}{q} \sqrt{\frac{n}{2(1-\varepsilon)}}\right)\right) d y,
\end{aligned}
$$

where $t=(1-y) t_{2}+y t_{1}$.

We can see that as a function of $|\nabla f|$, the quadratic

$$
\begin{aligned}
\rho|\nabla f| & -\left(t_{2}-t_{1}\right) \frac{|\nabla f|^{2}}{s}+\left(t_{2}-t_{1}\right) \frac{n s}{2(1-\varepsilon) t} \\
& +\left(t_{2}-t_{1}\right)\left(\frac{n s k}{2(1-\varepsilon)(s-1)}+\frac{c}{q} \sqrt{\frac{n}{2(1-\varepsilon)}}\right) \\
\leq & \frac{s \rho^{2}}{4\left(t_{2}-t_{1}\right)}+\left(t_{2}-t_{1}\right) \frac{n s}{2(1-\varepsilon) t}+\left(t_{2}-t_{1}\right)\left(\frac{n s k}{2(1-\varepsilon)(s-1)}+\frac{c}{q} \sqrt{\frac{n}{2(1-\varepsilon)}}\right) .
\end{aligned}
$$

So,

$$
\begin{aligned}
f\left(x_{1}, t_{1}\right)-f\left(x_{2}, t_{2}\right) \leq & \frac{s \rho^{2}}{4\left(t_{2}-t_{1}\right)}+\left(t_{2}-t_{1}\right)\left(\frac{n s k}{2(1-\varepsilon)(s-1)}+\frac{c}{q} \sqrt{\frac{n}{2(1-\varepsilon)}}\right) \\
& +\frac{n s}{2(1-\varepsilon)} \log \left(\frac{t_{2}}{t_{1}}\right)
\end{aligned}
$$

i.e.,

$$
\begin{aligned}
u\left(x_{1}, t_{1}\right) \leq & u\left(x_{2}, t_{2}\right)\left(\frac{t_{2}}{t_{1}}\right)^{\frac{n s}{2(1-\varepsilon)}} \\
& \times \exp \left(\frac{s r^{2}}{4\left(t_{2}-t_{1}\right)}+\left(t_{2}-t_{1}\right)\left(\frac{n s k}{2(1-\varepsilon)(s-1)}+\frac{c}{q} \sqrt{\frac{n}{2(1-\varepsilon)}}\right)\right) .
\end{aligned}
$$

\section{Conclusions}

In this paper, we use the method of gradient estimates to study the Fisher-KPP equation. We get the local gradient estimate (Theorem 1.1). Since the solution $u$ of (1.1) often describes the density, it is natural to study solutions of which $0<u<1$. We get the Harnack estimate if $0<u<1$ (Theorem 1.2). Our results can be used to study the solution of (1.1) further. The similar method can be also applied to the following equation:

$$
u_{t}=\Delta u+a u^{p}+b u^{q}
$$

where $a, b, p, q$ are constants. 


\section{Acknowledgements}

The authors would like to thank Professor Xiaodong Cao for his suggestion on the paper and would also like to thank referees for their valuable comments.

\section{Funding}

The second author was supported by the Chinese Universities Scientific Fund (2017LX003).

\section{Abbreviations}

Not applicable.

\section{Availability of data and materials}

Not applicable.

\section{Competing interests}

The authors declare that they have no competing interests.

\section{Authors' contributions}

All authors contributed equally to this work. All authors read and approved the final manuscript.

\section{Publisher's Note}

Springer Nature remains neutral with regard to jurisdictional claims in published maps and institutional affiliations.

\section{Received: 29 October 2017 Accepted: 21 February 2018 Published online: 27 February 2018}

\section{References}

1. Cao, X., Liu, B., Pendleton, I., Ward, A.: Differential Harnack estimates for Fisher's equation. Pac. J. Math. 290(2), 273-300 (2017)

2. Fisher, R.A.: The wave of advance of advantageous genes. Annu. Eugen. 7, 355-369 (1937)

3. Kolmogorov, A.N., Petrovsky, I.G., Piskunov, N.S.: Ėtude de l'équation de la diffusion avec croissance de la quantité de matière et son application à un problème biologique. Bull. Univ. Mosc. Sér. Int. A 1, 1-25 (1937). English transl. in: Pelcé, P. (ed.) Dynamics of Curved Fronts, pp. 105-130. Academic Press, New York (1988)

4. Hamel, F., Nadirashvili, N.: Travelling fronts and entire solutions of the Fisher-KPP equation in $\mathbb{R}^{n}$. Arch. Ration. Mech. Anal. 157(2), 91-163 (2001)

5. Berestycki, H., Hamel, F., Matano, H.: Bistable traveling waves around an obstacle. Commun. Pure Appl. Math. 62(6), 729-788 (2009)

6. Liang, X. Zhao, X.Q.: Asymptotic speeds of spread and traveling waves for monotone semiflows with applications Commun. Pure Appl. Math. 60(1), 1-40 (2007)

7. Cao, X.: Differential Harnack estimates for backward heat equations with potentials under the Ricci flow. J. Funct. Anal. 255(4), 1024-1038 (2008)

8. Cao, X., Hamilton, R.S.: Differential Harnack estimates for time-dependent heat equations with potentials. Geom. Funct. Anal. 19(4), 989-1000 (2009)

9. Li, P., Yau, S.T.: On the parabolic kernel of the Schrödinger operator. Acta Math. 156, 153-201 (1986)

10. Yau, S.T.: Harmonic functions on complete Riemannian manifolds. Commun. Pure Appl. Math. 28, 201-228 (1975)

11. Cheng, S.Y., Yau, S.T.: Differential equations on Riemannian manifolds and their geometric applications. Commun. Pure Appl. Math. 28(3), 333-354 (1975)

12. Li, J.: Gradient estimates and Harnack inequalities for nonlinear parabolic and nonlinear elliptic equations on Riemannian manifolds. J. Funct. Anal. 100, 233-256 (1991)

13. Negrin, E.R.: Gradient estimates and a Liouville type theorem for the Schrödinger operator. J. Funct. Anal. 127, 198-203 (1995)

14. Souplet, P., Zhang, Q.S.: Sharp gradient estimate and Yau's Liouville theorem for the heat equation on noncompact manifolds. Bull. Lond. Math. Soc. 38, 1045-1053 (2006)

15. Yang, Y: Gradient estimates for a nonlinear parabolic equation on Riemannian manifolds. Proc. Am. Math. Soc. 136 4095-4102 (2008)

16. Bailesteanu, M., Cao, X., Pulemotov, A.: Gradient estimates for the heat equation under the Ricci flow. J. Funct. Anal. 258(10), 3517-3542 (2013)

17. Bailesteanu, M.: Gradient estimates for the heat equation under the Ricci-harmonic map flow. Adv. Geom. 15(4) 445-454 (2015)

18. Albanese, G., Rigoli, M.: Lichnerowicz-type equations on complete manifolds. Adv. Nonlinear Anal. 5(3), 223-250 (2016)

19. Ghergu, M., Radulescu, V:: Nonlinear PDEs: Mathematical Models in Biology, Chemistry and Population Genetics. Springer Monographs in Mathematics. Springer, Heidelberg (2012)

20. Calabi, E.: An extension of E. Hopf's maximum principle with an application to Riemannian geometry. Duke Math. J. $25,45-56(1958)$ 